\title{
Esilio: patria delle rivelazioni in Maria Zambrano
}

\section{Exile: homeland of revelations in Maria Zambrano}

\author{
Andrea Cardinali*
}

Recebido: 31/08/2017. Aprovado: 05/12/2017.

Sommario: Il saggio intende mettere in luce la realtà dell'esilio vissuta e riscritta da Maria Zambrano, come momento di decostruzione e annientamento personali ma anche come luogo di rivelazioni uniche per scrivere e ripensare la storia. $\mathrm{Nel}$ pensiero della filosofa spagnola l'esperienza dell'esilio è fondante, è la pietra preziosa che custodisce i segreti di un'esistenza intera, uno spazio dove si rigenera la costante opportunità creativa di dare una speranza di unità al mondo.

Parole-chiave: Maria Zambrano. Esilio. Rivelazione.

Abstract: This paper aims to explore the reality of exile lived and rewritten by Maria Zambrano, as a moment of personal deconstruction and annihilation but also as a place of unique revelations to write and rethink history. For the Spanish philosopher the experience of exile is foundational, it is the precious stone that holds the secrets of a whole life, a space where the constant creative opportunity to give hope of unity to the world is regenerated.

Keywords: Maria Zambrano. Exile. Revelation.

\section{Introduzione}

In questo lavoro accenderemo un focus specifico sul luogo dell' esilio, in particolar modo prendendo in considerazione con metodo trasver-

* Giornalista e Scrittore. Dottore magistrale in Filosofia ed Etica delle Relazioni nell'Università degli studi di Perugia, Italia. Dottore magistrale in Ontologia Trinitaria nel Istituto Universitario Sophia, Incisa, Firenze, Italia. Ha pubblicato: "Sul dono di scrivere tra limite e mistero. In dia-logo con Flannery O'Connor" (Rivista Sophia, ricerche sui fondamenti e la correlazione dei saperi, Ann VII - n. 2, 2015). "Trucioli di mondo" (libro di narrativa di viaggio, collana Cassiciacum, 2015)

E-mail: andicardi@hotmail.it 
sale alcuni dei più importanti testi di Maria Zambrano, filosofa spagnola che fu costretta a rimanere lontana dalla patria per oltre quarant'anni.

Nella prima parte affronteremo la questione dell'esilio osservando da vicino la condizione dell'esiliato: la particolare situazione di sofferenza e sospensione che vive sia da un punto di vista spazio-temporale, che esistenziale.

La seconda parte si concentrerà sul rapporto tra patria ed esilio. Tenteremo di mostrare, ripercorrendo le parole dell' autrice, come questo tempo forzato di lontananza, pur nella sofferenza, può divenire uno spazio privilegiato attraverso il quale, non solo vedere la patria natale sotto una diversa luce ma scoprirsi parte essenziale di essa proprio perché nella condizione di esiliato. Proveremo a seguire la pensatrice andalusa nel difficile passaggio di comprensione di quelle che potrebbero definirsi le sue parole più celebri e paradossali: "Amo il mio esilio» ${ }^{1}$ e che portano la realtà dell'esilio a identificarsi come patria. Proveremo a entrare in questa "identificazione" e mettere in luce il ruolo che la scrittura occupa all'interno del pensiero dell'autrice di Velez-Malaga.

La terza e ultima parte vorrà guardare all'esilio come una realtà nella quale accadono importanti rivelazioni soprattutto per mezzo di due elementi significativi che si richiamano a vicenda: la ferita che l'esiliato patisce, e la diversità con la quale è costretto a incontrarsi e relazionarsi ogni volta. Proveremo in questa fase a tracciare una via che possa accompagnare il pensiero illuminante e la scrittura intuitiva della Zambrano nella direzione di un orizzonte culturale più ampio, verso il quale ella si orientava, affinché il «pensiero venga assimilato e rinasca, come rinasce tante volte quante sono le generazioni che riempiono il tempo della storia» ${ }^{2}$.

\section{La condizione dell'esiliato}

Maria Zambrano appartiene a quella cerchia di intellettuali che, a causa della guerra civile ${ }^{3}$, furono costretti a scegliere tra rimanere in patria vivendo un "silenzio intellettuale" oppure l'esilio. Ella scelse quest'ultimo, intuendo che restare in Spagna avrebbe significato vivere

1 ZAMBRANO, M. Le parole del ritorno. Enna: Città Aperta, 2003. p. 24.

2 ZAMBRANO, M. Verso un sapere dell'anima. Milano: Raffaello Cortina, 1996. p. 26.

$3 \quad$ Nel caso dell'autrice parliamo della guerra civile spagnola esplosa nel 1936. 
ogni giorno in un ambiente ostile e pericoloso, data la propria partecipazione al Governo della Resistenza. Negli scritti della filosofa troviamo vari cenni sulla generazione alla quale apparteneva, che ella chiama "generazione del toro per il suo significato sacrificale: esseri molto cari, vittime" 4 . La maggior parte dei simpatizzanti repubblicani non ebbero infatti neppure il tempo di scegliere tra le due "alternative" e vennero uccisi. Proprio a loro, la Zambrano si rivolge in Delirio e Destino con queste sofferenti parole: «voi, i morti, lasciati senza tempo; noi, $i$ superstiti, lasciati senza luogo» ${ }^{5}$.

Nella parabola filosofica della Zambrano l'espressione "senza luogo" è utilizzata per parlare in prima persona dell'esilio e, più in generale, come immagine di coloro che sono stati esiliati scegliendo una strada diversa, senza nascondersi dietro a giustificazioni alcune. Nella Lettera sull'esilio scrive:

Il nostro silenzio, il silenzio degli esiliati, che hanno parlato così poco dell'esilio mentre avrebbero potuto parlarne tanto, dimostra che non si è seguita la via della giustificazione, per la quale si sfila armati di risplendenti ragioni, ma l'altra, che neppure sembra una via: quella di andarsi spogliando di torti e anche di ragioni, di volontà e di progetti. Andarsi spogliando sempre più di tutte queste cose per rimanere nudi e distaccati: soli e immersi in se stessi, e insieme alle intemperie, come uno che sta nascendo; nascendo e morendo al tempo stesso, mentre la vita continua. La vita che lasciarono all'esiliato senza ch'egli ne avesse colpa. Tutta la vita e il mondo, ma senza un luogo in esso, dovendo vivere senza veramente stare, cosa tanto necessaria. Muoversi senza poter quasi agire, ed essere così allo stesso tempo colui che dimora in una caverna, come chi nasce, e nel deserto, come chi muore. ${ }^{6}$

Pochi autori hanno studiato e riflettuto tanto profondamente sulla particolare condizione dell'esilio come la filosofa di Malaga. La novità principale non sta nella condizione esiliare che l'ha costretta a un periodo di quarantacinque anni fuori dalla patria ${ }^{7}$, situazione che storicamente, come ricordato, è toccata ad altri come lei. Ciò che la rende la "filosofa

\footnotetext{
ZAMBRANO, M. Le parole del ritorno. Enna: Città Aperta, 2003. p. 59.

ZAMBRANO, M. Delirio y destino. Madrid: Mondadori, 1989. p. 209.

6 Lettera pubblicata per la prima volta in Cuadernos del Congreso por la libertad de la cultura, 49 (1961), pp. 65-70 Cfr. ZAMBRANO, M. L'esilio come patria. Brescia: Morcelliana, 2016. p. 77.

7 Dal 1939 al 1984.
} 
dell'esilio" è il suo studio approfondito, la sua attenta analisi storica ed esistenziale del dramma che l'esiliato vive. La forza sta nella forma che riesce a dare al pensiero, senza mai allontanarlo dall'esperienza reale vissuta, della quale raccoglie e riordina i tratti più nascosti attraverso la propria personalità. Da pensatrice esiliata, diventa così, nel lungo tempo passato fuori dalla Spagna, una delle più grandi teoriche dell'esilio, in parte per necessità dovendolo accettare e vivere, in parte per la sua straordinaria capacità di restare coerente con il proprio sistema filosofico che trova la spinta propulsiva da ciò che le sta attorno, da un sempre nuovo e personale incontro con il mondo: "Ogni esperienza ha qualcosa di rivelato per quanto si dia nella relatività dell'umano". Scrive l'autrice in Senderos. "Proprio in quanto relativo, l'uomo ha bisogno della rivelazione delle verità che sono intorno a noi finché non le si rivive. Esperienza è rivelazione e storia" ${ }^{\text {. }}$

Queste parole precisano senza mezzi termini che compiere uno studio sul pensiero della Zambrano equivale a penetrare nella sua esistenza, nel dramma dell'esilio, muoversi sull' affascinante frontiera della razòn poetica, in una stimolante ricerca interdisciplinare tra realtà storica e filosofia, cercando di entrare nel suo originale linguaggio che ricorre spesso a immagini, espressioni poetiche e figure retoriche.

L'esilio, prima di essere riconosciuto dall'autrice come luogo in cui accadono importanti rivelazioni, deve però essere riconosciuto come esilio, cioè come sofferenza, come punto di rottura e di abbandono. Scrive Zambrano:

Comincia, l'iniziazione all 'esilio, quando comincia l'abbandono, il sentirsi abbandonato. [...]. E qui comincia l'esilio, il sentirsi ormai sull'orlo dell'esilio. [...]. Nell'abbandono, solo quel proprio di cui si è spossessati appare, solo ciò che non si può giungere a essere come essere proprio. Il proprio c'è soltanto in quanto negazione, impossibilità. Impossibilità di vivere che, quando ci si rende conto di questo, è impossibilità di morire ${ }^{9}$.

L'esilio è la terra dell'impossibilità, lo spazio impossibile nel quale vive un uomo impossibile, colui che è stato cacciato dalla sua vita e lasciato fuori da sé. Nell'abbandono, l'esiliato può vedere soltanto le sue mancanze, il proprio essere divenuto inesistente, senza qualità. E pur pensando di morire e forse sperandolo, si trova invece provvisto

8 ZAMBRANO, M. Senderos. Barcellona: Anthropos, 1986. p. 23.

9 ZAMBRANO, M. I Beati, Milano: SE SRL, 2010. pp. 31-32. 
anche di questo tipo di impossibilità. L'unica cosa che vede, scrive la Zambrano, è una "linea di demarcazione tra vita e morte che in egual modo si respingono". E continua l'autrice: "Mantenersi su questa linea è la prima esigenza che all'esiliato si presenta come ineludibile”10. La condanna dell'esiliato è quella di trattenersi per un tempo indeterminato in uno stato di costante indeterminatezza. Questa è la condizione che più di ogni altra lo caratterizza: "non avere un posto nel mondo", scrive la Zambrano "né geografico, né sociale, né politico, né [...] ontologico" 1 .

La maledizione di vivere in questo luogo ostile di solitudine, abbandono e impossibilità della vita di patria, se in una prima fase getta la pensatrice nell'umano sconforto, la coglie poi in una rinascita. Nel Manoscritto M.157 conservato a Velez-Malaga presso la Fundaciòn a lei intitolata, Maria Zambrano traccia un rapporto di similitudine tra l'esilio e la nascita, poiché "vivere è 'entrare in', in una 'situazione' che l'esiliato, al tempo in cui esce, deve procurarsi"; il che presuppone di

uscire dal tutto in cui si era, di uscire dalla situazione in cui si viveva, il che equivale ad uscire dalla vita determinata dove si è qualcuno da qualche parte. [...]. Uscire del tutto in quell 'istante e quell' istante seguirà sempre l'esiliato, come se fosse nessuno (nadie), esattamente neanche uno (ninguno). [...]. Nessuno, neanche uno (nadie, ninguno), poiché basta che la situazione ove siamo qualcuno svanisca, e noi rimaniamo soli dinanzi alla vita tutta, con la vita, affinché sentiamo di essere nessuno, nessuno, come se il soggetto perdesse la sua determinazione immediata, tranne l'assoluto che gli si offre. Come colui che nasce ${ }^{12}$.

L'esperienza abissale di questa forzata lontananza permette alla filosofa di penetrare i luoghi più intimi del proprio essere, attraversando varie complicate fasi che la mettono in ricerca di un nuovo equilibrio esistenziale. Il trovarsi nella condizione di "colui che nasce" o che va verso una nuova nascita, significa abitare il travaglio del non essere ancora nato, di passare in lungo e in largo nel territorio del nadie, dell'essere nessuno. Armando Savignano spiega questo importante passaggio, citando nel suo intervento alcune parole di Emmanuel Lévinas. Scrive il filosofo italiano:

\footnotetext{
10 Ivi, p. 32.

11 Ivi, p. 35

12 ZAMBRANO, M. Manoscritto M.157, conservato presso la Fundaciòn Maria Zambrano.
} 
L'esiliato ha attraversato la dis-nascita e la de-creazione, sottraendosi all'impulso a esistere; è aperto ad una nuova nascita, una nascita continua, quella creata proprio grazie all'esilio: essere continuamente in cammino verso se stessi e la propria trascendenza. Cosi emerge l'estrema vulnerabilità e l'abbandono totale, l'incolmabile distanza dagli altri e pertanto la grande solitudine. Si può dire che l'essere dell'esiliato è dunque quello 'dell'esistenza separata dall'esistente ${ }^{13}{ }^{\prime \prime 14}$.

La questione dell'esilio pone l'uomo al centro di un paradosso esistenziale che "lo scaglia" in un'esperienza di crisi, nella quale si fa presente costantemente l'assenza di luogo. Scrive Zambrano:

Di sradicamento in sradicamento, in ciascuno di essi l'esiliato va morendo, spossessandosi, sradicandosi. E cosi riprende ad andare, si reitera la sua partenza dal luogo di origine, dalla sua patria e da ogni possibile patria, perdendo egli a volte il poco che ha nel fuggire la seduzione di una patria che gli si offre, correndo davanti alla sua ombra tentatrice; nel qual caso viene inevitabilmente accusato di questo, di andarsene, di andarsene senza sapere nemmeno dove. Perché ciò da cui fugge chi all'esilio è promesso, marcato da esso sin da prima, è un dove, un luogo che sia il suo ${ }^{15}$.

L'esiliato è a tal punto "spogliato di sé" che non può fermarsi in nessun luogo, è come un viandante senza meta che nel profondo conserva l'unica patria originaria ma che, allo stesso tempo, vive esperienze circoscritte che lo avvicinano a pensare di poter trovare una possibile nuova patria. La tentazione di un luogo che lo faccia uscire dal territorio dell'impossibilità in cui è, viene però fatalmente vinta nel preciso momento in cui sente una "tacita promessa", già da prima esistente, che lo risveglia dal "sogno seduttore" di "un luogo che sia il suo." Egli, nelle accuse che soffre andandosene ogni volta da una "possibile patria", compie, ricevendolo, un nuovo sradicamento per ricominciare l'esperienza di un "esilio minore" nel "grande esilio" che gli è toccato. Così commenta la filosofa:

E, nel frattempo, colui che si è ritrovato solo all'ombra immensa della vulnerabilità estrema dinanzi all'immensità della vita, senza nemmeno sentire che la vita procede in quest'immensità, è rimasto così, semplice-

13 LÉVINAS, E. Dall'esistenza all'esistente. Genova: Marietti, 2000.

14 ZAMBRANO, M. L'esilio come patria. Brescia: Morcelliana, 2016. p. 13.

15 ZAMBRANO, M. I Beati. Milano: SE SRL, 2010. p. 36. 
mente così, non saprebbe dire come né perché, né quale sia il punto di partenza dello strappo forzato da ciò che fu patria, città, casa, orizzonte, paesaggio familiare. Egli cessa propriamente di essere sradicato per avviarsi a essere un esiliato ${ }^{16}$.

Una delle immagini più eloquenti che la Zambrano utilizza per cercare di descrivere il luogo dell'esiliato, a conferma del suo intermittente ma incessante andarsene creando "esili minori", è l'immagine delle isole. Scrive la filosofa spagnola:

Le isole, luogo proprio dell'esiliato che le fa senza saperlo là dove non compaiono. Le fa o le rivela lasciandole galleggiare nell'illimitatezza delle acque posate su di esse, sostenute dal respiro che viene da remote lontananze, perfino dallo stesso firmamento, dallo scintillare delle sue stelle, mosse esse da invisibile brezza ${ }^{17}$.

Costui è nella condizione "propria di chi abbandona la terrafer$m a$ "18, per dirla con un'espressione del filosofo italiano Cacciari, e si inoltra in mare aperto senza una precisa direzione. L'esiliato, trovandosi in un ambiente dalla natura fluida, in movimento continuo ed esposto ai pericoli, può solo cercare di creare delle "isole", punti di attracco che galleggiano nello spazio e nel tempo e che stanno al di fuori della sicurezza terrena che contraddistingue la patria.

Maria Zambrano sottolinea più volte come l'esilio, gettando l'uomo fuori dalla patria, lo escluda anche dalla categoria storica, sospendendolo in una dimensione che sta fuori. "La Patria", scrive la pensatrice andalusa, "è una categoria storica, non così la terra, né il luogo. La Patria è luogo di storia, terra dove un giorno fu seminata una storia la cui crescita è stata calpestata più di quella di qualsiasi altra" 19 .

Per la pensatrice, essendo la patria un luogo in cui "fu seminata la storia", nel suo nascere e presentarsi a questa, crea inevitabilmente l'esilio. Scrive:

Possiede, la patria vera, la virtù di creare l'esilio. È il suo segno inequivoco. E così, non appena albeggia nella storia, non appena si dà minimamente a vedere - basta in verità che si annunci - essa crea

16 Ivi, p. 39

17 Ivi, p. 40.

18 CACCIARI, M. L'arcipelago. Milano: Adelphi, 1997. p. 28.

19 ZAMBRANO, M. I Beati. Milano: SE SRL, 2010. p. 35. 
l'esilio di quanti, per averla anche minimamente vista e servita, devono allontanarsene ${ }^{20}$.

L'esperienza dell'esilio nel suo stato più profondo, pur al di fuori della storia, diventa per la Zambrano necessaria ed essenziale per scoprire la patria.

La lontananza e la totale assenza di questa originaria "terraferma" giunge a un punto di soglia nell'esiliato, che genera in sé una sorta di quiete, di rassegnazione nei suoi confronti. In quel preciso istante, quando nessuna terra sembra appaia all'orizzonte, ecco rivelarsi la patria. Scrive la pensatrice:

L'esilio è il luogo privilegiato per lo scoprirsi della Patria, perché essa stessa si scopra quando l'esiliato ha ormai smesso di cercarla. Ormai senza più sete, il suo sguardo non la intravede nel vuoto lasciato dall'ultimo raggio di sole, né nell'albero caduto che si ostina a rinverdire, [...] né in nessun 'altra parte. Quando ormai si sa senza di essa, senza alcuna sofferenza, quando ormai non si riceve nulla, nulla dalla patria, allora essa gli appare. Non la può definire, dato che nemmeno la riconosce ${ }^{21}$.

\section{L'esilio come patria}

Parlare dell'esilio come patria sembra, e a tutti gli effetti lo è, un grande paradosso. Maria Zambrano abitando l'esilio per quarantacinque anni e scrivendo della grande sofferenza che prova sulla sua pelle in tante piccole e grandi situazioni ordinarie, non si dimentica però anche di vedere il proprio cambiamento da una prospettiva di crescita personale, andando a sviscerare ciò che questa specifica condizione di estrema vulnerabilità può procurare in termini di arricchimento del pensiero.

L'elemento che forse più di ogni altro fa riflettere sull'esilio come luogo di pensiero e rivelazione, è proprio ciò che in tale condizione appare mancante: la patria. Se infatti le argomentazioni teoriche sull'esilio, che derivano dall'esperienza vissuta, ci raccontano lo stare fuori dalla patria come condizione da accettare e far propria, è vero anche che il ricordo della Spagna riporta a galla, nella pensatrice andalusa, la vita di contrasti e prese di posizioni politiche, i soprusi subiti negli anni della guerra da

$20 \quad$ Ivi, p. 41.

21 Ibidem. 
parte del popolo spagnolo e l'imposizione per mezzo di violenze del regime franchista. Tutto questo, sommato all'esilio toccatole personalmente, apre una nuova breccia nelle parole dell'autrice che, proprio come ci suggerisce nei suoi scritti, ripensa dal suo esilio il concetto di patria "perché la patria sia patria e non un luogo 'occupato' da coloro che giungono in virtù della forza oppure in virtù della forza dell'età "22.

L'esiliato è rimasto senza luogo, non ha un luogo preciso verso cui dirigersi ma l'essere costantemente aperto alle "intemperie del mondo" e della gente, lo mette allo stesso tempo nella condizione di dover cercare sempre qualcosa, di non perdere mai la speranza e di continuare a tenere in movimento e in trasformazione il proprio pensiero, a differenza di coloro che vivono in patria. Riguardo a loro la pensatrice scrive:

L'esiliato è stato lasciato senza nulla, al margine della storia. [...] Essi invece, con un luogo, ma in una storia priva di antefatti. Dunque anch'essi senza luogo; senza luogo storico. [...] Sono rimasti senza orizzonte. E per quanto stiano nella terra, nella loro, dove si parla il loro idioma, dove possono dire: 'Sono cittadino', quando rimane senza orizzonte, l'uomo, animale storico, perde anche il luogo, in ciò che si riferisce alla storia. Non sa quel che gli accade, non sa quel che sta vivendo. Vive in un sogno.

Con questa differenza, che l'esiliato ha dovuto destarsi. E se è rimasto così, assorto in se stesso e come estraneo a tutto, anche alla sua stessa storia, è perché la vede, perché la contempla con sempre maggiore chiarezza e precisione, da quel luogo, da quel limite tra la vita e la morte dove abita, luogo privilegiato perché si determini la lucidità, soprattutto quando si è rinunciato a giustificarsi e non si è ceduto alla tentazione di cristallizzarsi in un personaggio; quando non si è voluto essere nulla, neppure un eroe ${ }^{23}$.

Questo scritto si trova nella Lettera sull'esilio pubblicata per la prima volta nel 1961, esattamente nel cuore dell' esilio di Maria Zambrano, che sarebbe terminato ufficialmente con il suo ritorno in Spagna nel 1984. Da questo testo in poi, la prospettiva dell'autrice, pur rimanendo in linea con quanto trascritto precedentemente riguardo la sua comprensione dell'esilio, schiude in lei una nuova "fessura ermeneutica", attraverso la

22 ZAMBRANO, M. L'esilio come patria. Brescia: Morcelliana, 2016. p. 84

23 Ivi, p. 85. 
quale poter guardare all'esilio e alla patria in una più ampia complessità, con uno sguardo più integrale. Scrive la filosofa:

Coloro che sono rimasti e cresciuti in patria [...] rimangono nel loro sogno; con la realtà, sì, ma una realtà che si offre loro come sognata perché slegata dal suo ieri, chiusa in se stessa, privata dell'orizzonte. Mentre all'esiliato è rimasto quasi soltanto orizzonte; orizzonte senza realtà, orizzonte nel quale egli guarda, passa e ripassa, sgrana la storia, tutta la storia, soprattutto la storia di Spagna.

A questo, egli è stato obbligato: a passare e ripassare la storia della sua patria, giacché gli hanno domandato conto di essa per tutte le strade del mondo ${ }^{24}$.

La paradossale situazione che la filosofa spagnola ci descrive in maniera così trasparente, è quella di una "patria di esiliati", coloro cioè che pur vivendo in patria o meglio, proprio perché vivono in patria, soffrono inconsapevolmente "l'esilio dell'orizzonte", cioè sono privati di quello sguardo che può andare oltre la loro realtà slacciata dalla storia passata e chiusa in uno spazio ristretto e addormentato. Per questo motivo l'autrice scrive che è una "realtà sognata", un sogno a occhi chiusi che blocca l'apertura dello sguardo sulla propria condizione presente e sul tempo che verrà.

All'esiliato, invece, è rimasto solo questo sguardo, attraverso il quale rivedere la storia e con essa la patria, per provare a comprenderla e risponderne, dato che "per tutte le strade del mondo" gli viene in qualche maniera ricordato che ne è, seppur in maniera parziale, una rappresentazione. Scrive la Zambrano:

$E$ ha dovuto render conto di tutto; s'è visto investito della categoria di rappresentante della storia della sua patria, e siccome sentiva la patria, siccome non poteva lasciarla abbandonata alle opinioni della gente, ai luoghi comuni; siccome accettava la sua eredità, [...] ha dovuto entrare nelle viscere di questa storia, è vissuto nei suoi inferni; più volte è disceso in essi per uscirne con un poco di verità, con una parola di verità strappata da essi. Ha dovuto a poco a poco trasformarsi, senza rendersene conto, in coscienza della storia ${ }^{25}$.

$24 \quad$ Ivi, p. 86.

25 Ibidem. 
L'esilio, senza perdere i connotati della condizione di sofferenza e disagio che provoca in chi lo vive, comincia a diventare, per la filosofa, uno spazio di incontro con la verità storica, con il mondo e con il proprio essere nel mondo.

$\mathrm{Al}$ ritorno in Spagna, riuscirà a ripercorrere il suo esilio visto da una nuova prospettiva: staccandosi e distinguendosi da esso non potrà che parlarne e scriverne con grande sentimento, con l'affetto che, appunto, si nutre per la propria patria. Così scrive:

Di certi viaggi si comincia a sapere solo al ritorno. Per me, guardato dalla prospettiva del ritorno, l'esilio che mi è toccato vivere è essenziale. Io non concepisco la mia vita senza l'esilio che ho vissuto. L'esilio è stato la mia patria, o come la dimensione di una patria sconosciuta che, una volta conosciuta, diventa irrinunciabile ${ }^{26}$.

L'esperienza dell'esilio ha segnato profondamente la vita di Maria Zambrano, tanto da diventare un luogo irrinunciabile per lo sviluppo del suo pensiero filosofico. La maggior parte dei suoi lavori sono stati scritti nell'arco di quei quarantacinque anni, un tempo ostile che pur non accogliendola, l'ha però cresciuta, fatta nascere di nuovo, fondando in buona parte il suo pensiero. Lei stessa ce lo racconta nello scritto Amo il mio esilio raccolto ne Le parole del ritorno:

Credo che l'esilio sia una dimensione essenziale della vita umana, ma nel dirlo mi mordo le labbra, perché vorrei che non ci fossero mai più esiliati, che tutti fossimo a un tempo esseri umani e cosmici, che l'esilio fosse sconosciuto. È una contraddizione, cosa posso farci; amo il mio esilio, forse perché non lo ho cercato, perché non sono stata io a inseguirlo. No, l'ho accettato piuttosto; e quando si accetta qualcosa di cuore, perché sì, costa molto rinunciarvi.

Io ho rinunciato al mio esilio e sono felice, sono contenta, ma questo non me lo fa dimenticare, sarebbe come rinnegare una parte della nostra storia e della mia storia. I quarant'anni di esilio non può ridarmeli nessuno, il che rende più bella l'assenza di rancore. Ho ormai accettato pienamente il mio esilio ma, nello stesso tempo, non chiedo ne auguro ad alcun giovane che lo comprenda, perché per comprenderlo dovrebbe soffrirlo, e io non posso augurare a nessuno di essere crocefisso ${ }^{27}$.

26 ZAMBRANO, M. Le parole del ritorno. Enna: Città Aperta, 2003. p. 24. 


\section{Le rivelazioni dell'esilio: ferita e diversita'}

"Risulterà eccessivo questo termine 'rivelazione', applicato all'esilio?" Si domanda Maria Zambrano, che immediatamente risponde: "Il rischio c'è, quando l'idea che qualcosa ci sia stato rivelato viene costantemente respinta. La rivelazione è stata ristretta allo specificamente religioso" ${ }^{\prime 28}$.

Una delle caratteristiche peculiari nella scrittura della Zambrano è il voler recuperare le parole, salvare in loro la "forza originaria", affievolita negli anni da una sistematica circoscrizione per ambito o disciplina. Per la pensatrice andalusa la parola "rivelazione" svolge una funzione essenziale perché le permette di dare un nome a ciò che va scoprendo durante la dolorosa e misteriosa esperienza dell'esilio.

"Ed è nell'essere e a partire dall'essere che si ricevono le rivelazioni. È la visione a darsi all'essere. Una teoria della conoscenza della rivelazione si fa ogni giorno più necessaria "29 ${ }^{2}$ scrive l'autrice, che poco più avanti precisa:

L'esperienza è a partire da un essere, questo che è l'uomo, questo che sono, che vado essendo io in virtù di ciò che vedo e patisco e non di ciò che ragiono e penso. Perché l'uomo patisce se stesso e attraverso ciò che vede. Ciò che vede lo ferisce, lo può ancora ferire prodigiosamente perché il suo essere gli si apra e gli si riveli" ${ }^{\prime 30}$.

In questa visione, che fonda la razòn poetica, il vedere e il patire si fanno immagini e metafore per poter andare a creare nel lettore un possibile spazio di rivelazione. Se come scrive la filosofa spagnola, il vedere può ancora "ferire prodigiosamente" e portare alla rivelazione, significa che molto spesso le più grandi rivelazioni giungono dal dolore, da un tempo di sofferenza o un momento drammatico che l'uomo vive.

Una delle più grandi scrittrici americane del ventesimo secolo, Flannery O'Connor, nel celebre testo Nel territorio del diavolo, sul mistero di scrivere, racconta la "funzione rivelatrice" della sua narrativa che si basa sull'azione violenta della "grazia" che "sveglia" in maniera brutale i personaggi chiusi nella loro mentalità. Il lettore, "vedendo" e

\footnotetext{
28 ZAMBRANO, M. I Beati. Milano: SE SRL, 2010. p. 29.

29 Ibidem.

30 Ivi, p. 30.
} 
"toccando" la ferita del personaggio, è portato a "sentirla" nelle azioni della propria vita e compiere un cambiamento radicale di prospettiva. Scrive Flannery O'Connor:

Suppongo che le ragioni per l'impiego di tanta violenza nella narrativa moderna differiscano da scrittore a scrittore, ma nei miei racconti ho riscontrato che essa è stranamente capace di ricondurre i personaggi alla realtà e di prepararli ad accettare il loro momento di grazia ${ }^{31}$.

Zambrano descrivendo l'esperienza drammatica dell'esilio, non rivela le verità dicendole, ma mostrandole. Non è infatti nello "spiegare", ma nel mostrare che il lettore può scoprire ed entrare nel "segreto" svelatogli da colui che scrive. Anche la narrativa di Flannery O'Connor segue questa linea performativa. Afferma l'autrice americana:

Se non gli viene dato modo di vivere la storia, di toccarla con mano, il lettore non crederà a niente di quello che il narratore si limita a riferirgli. [...] Lo scrittore di narrativa deve rendersi conto che non è possibile suscitare la compassione con la compassione, l'emozione con l'emozione, o i pensieri con i pensieri. A tutte queste cose bisogna dar corpo, creare un mondo dotato di peso e di spessore ${ }^{32}$.

Pur parlando di strumenti e generi diversi di scrittura, vediamo come in Maria Zambrano e in Flannery O'Connor ci sia la stessa "vocazione intellettuale", la propensione, cioè, a creare una relazione con il lettore che possa mettere insieme l'aspetto razionale con quello rivelativo per mezzo di quello letterario-performativo.

Guardando all'esilio di Maria Zambrano come spazio di rivelazione e con la prospettiva da lei auspicata di un cambiamento culturale, assieme all'esperienza della ferita vi è quella importante della diversità.

La pensatrice andalusa vive in prima persona la condizione dell'esistere fuori dalla patria, dell'essere guardata ogni volta come "la straniera", "la sconosciuta". Abitando così a lungo questo status, l'elemento della diversità non può che considerarsi in lei come sostanziale per le rivelazioni. "L'esiliato è colui che più assomiglia allo sconosciuto, colui che", continua la Zambrano "a forza di portare all'estremo la sua condizione, arriva a essere quello sconosciuto che c'è in ogni uomo e

31 O'CONNOR, FI. Nel territorio del diavolo, sul mistero di scrivere. Roma: Minimun Fax, 2010. p. 75.

32 Ivi, pp. 58-60. 
che il poeta e l'artista non riescono se non molto raramente a scoprire". ${ }^{33}$ L'esilio appare allora come luogo privilegiato per cogliere la parte sconosciuta dell'essere umano, per entrare in relazione con la diversità più profonda, essendo essa radicata e riconosciuta personalmente da colui che vive l'esilio.

La filosofa comprende che esistono elementi di grande "ricchezza rivelatrice" in culture molto diverse da quella occidentale e che, se l'Occidente continuerà a non prenderle in considerazione o peggio a "categorizzarle" all'interno dei propri sistemi, perderà una grande opportunità di scoperta e trasformazione. Così scrive:

Fortunatamente, le investigazioni di altre storie, di altre culture viventi o sepolte dal tempo, l'archeologia stessa, la filosofia, la storia delle religioni soprattutto, offrono conoscenze e ancor più indizi, barlumi di visioni non riducibili alle analisi, rivelazioni, dunque. Ma rivelazioni che saltano da sole nel recinto della ragione occidentale. Che rimangono rinchiuse dentro le categorie vigenti: situazione, circostanza... tutte sottostanti alla categoria suprema dell'esplicabile - e, se si tratta di una vita umana del giustificabile. A ogni rivelazione tocca giustificarsi, tocca provare il suo diritto di cittadinanz $a^{34}$.

L'accusa che qui muove la filosofa è riguardo a un sistema di pensiero, quello dell'Occidente, che tende ordinariamente a inglobare e ridurre in analisi le rivelazioni storiche, religiose, culturali, sterilizzandone la forza o tentando di farlo. Le rivelazioni sono delle diversità che sobbalzano, che lasciano una traccia luminosa o un indizio sul sentiero della storia, "barlumi di visioni" che l'uomo deve poter cogliere per rinnovare il proprio pensiero.

Sébastien Jallade, antropologo statunitense descrive così questo tipo di situazione che può portare a pericolose conseguenze:

La pretesa delle nostre società di quantificare tutto comporta una conseguenza pericolosa: la perdita di ciò che è sconosciuto. Se non ci si prende cura di questo, ci sarà, dietro la visione quantitativa del reale, un'assenza lampante di umanità per descrivere il mondo che noi viviamo e i segreti che devono abitarlo. Quindi ogni testimonianza che portiamo

33 ZAMBRANO, M. I Beati. Milano: SE SRL, 2010. p. 34.

34 Ivi, p. 29. 
con noi, per quanto imperfetta sia, conserva una parte di mistero. Da questa verità non svelata, preservata, nasce il sacro. ${ }^{35}$

Nel "mondo quantificato" che ci descrive Jallade vengono a smarrirsi quelli che sono i valori fondanti dell'essere umano, il quale corre il rischio di pensare di aver raggiunto il potere di misurare e definire ogni cosa che gli sta attorno. La perdita "del senso del mistero" che fin dall'inizio dei tempi ha interpellato l'umanità sul suo scopo e su quello dell'universo, corrisponde alla perdita della domanda sul senso dell'esistenza e sulla crisi della dimensione interiore della persona.

Zambrano in Verso un sapere dell'anima affronta questa problematica:

È inevitabile accettare il fatto che, se consideriamo quest'ultimo periodo, lo troviamo pieno di scienza e conoscenza pura: di conoscenza applicata alla tecnica e alla fabbricazione di strumenti, ma povero, immensamente povero di tutte le forme attive della conoscenza. E per attive intendiamo quelle che nascono dal desiderio di penetrare nel cuore umano, quelle che si fanno carico di diffondere le idee fondamentali per utilizzarle come ispiratrici nella vita quotidiana dell'uomo comune che non è, né pretende essere filosofo o saggio ${ }^{36}$.

Ricomprendere i ruoli dell'esiliato, dello straniero, del rifugiato, dello sconosciuto, come "diversità" che "ferendo" la nostra cultura, la arricchisce di una storia e di un orizzonte, appare essenziale per poter pensare una rinascita culturale di quest'epoca.

Chiara Lubich pensatrice e mistica, fondatrice del Movimento dei Focolari, coniò negli anni '70 l'espressione di Uomo-Mondo che descrive in maniera calzante questo tipo di atteggiamento di apertura alla diversità.

Con questo discorso si rivolse alle giovani del Movimento dei Focolari il 22 luglio 1972:

Il mondo di oggi sta scoprendosi reciprocamente attraverso i mezzi di comunicazione sociale, per cui certi popoli che per noi erano completamente sconosciuti, certe religioni che noi non conoscevamo, adesso attraverso la radio, la stampa, il cinema, attraverso soprattutto la tele-

35 JALLADE, S. I/ richiamo della strada. Portogruaro: Ediciclo, 2011. p. 60.

ZAMBRANO, M. Verso un sapere dell'anima. Milano: Raffaello Cortina, 1996. p. 55. 
visione, incominciamo a conoscere questi altri popoli, che prima erano lontanissimi da noi.

Siamo quindi nell'epoca in cui è necessario che i giovani si formino con una mentalità non più soltanto occidentale, o mentalità musulmana, o mentalità buddista, o mentalità orientale, ma siamo nel tempo nel quale i giovani debbono formarsi con una mentalità mondo.

Alcuni anni più tardi, il 10 maggio 1997, durante la manifestazione internazionale Supercongresso Gen 3, Chiara Lubich, riprendendo questa definizione, affermava:

Ecco, l'uomo di domani, la persona di domani è l'uomo dell'unità, l'uomo-mondo. Un uomo che riesce ad accogliere nel suo cuore i tesori che donano gli altri dei vari continenti e che riesce a dare $i$ suoi tesori a tutti gli altri. L'uomo di domani, perciò, è l'uomo dell'unità, è l'uomo-mondo.

La pensatrice italiana definisce così l'uomo del domani, un uomo che nella diversità sappia generare unità, accogliendo " $i$ tesori" degli altri continenti e donando ciò che di più prezioso ha ricevuto dalla propria cultura. La persona che si apre a questa maniera di vivere è di una nuova umiltà perché accesa fortemente dal desiderio di imparare da tutti. La Lubich scrive che, nell'incontro con le culture diverse dalla propria, gli uomini cominciano ad accostarsi non più "in veste di colonizzatori che vanno ad imporre il proprio modo di vedere il mondo e la vita”.

E continua:

Solo adesso cominciamo ad avvicinare quei popoli col dovuto rispetto, con l'umiltà di chi sa che deve sempre imparare e per questo ci accorgiamo che c'è tutto un altro modo di vedere le stesse cose, diversissimo dal nostro, di noi occidentali, ma non per questo meno giusto o meno valido.

Tutti noi, chi più chi meno infatti, siamo cresciuti alla scuola della mentalità greca e romana. Socrate, Platone, Aristotele, Cicerone, Tacito, Giustiniano sono stati i nostri maestri di pensiero. Il linguaggio, il vocabolario che adoperiamo è stato nella più gran parte, formato da loro. Non solo: ma da noi nell'epoca attuale domina una cultura tecnologica. Ebbene, ora siamo di fronte a popoli che non sanno nulla della Grecia, di Roma, della nostra mentalità, ma poggiano le loro grandi civiltà su altri nomi, su altri pensatori grandi pure loro e a noi sconosciuti. ${ }^{37}$

37 LUBICH, Ch. Colloqui con i gen. Roma: Città Nuova, 1974. pp. 168-169. 
La globalizzazione e lo sviluppo tecnologico hanno generato nell'uomo di oggi reazioni opposte: da una parte il perentorio radicamento nella propria cultura con barriere di separazione al confine degli stati, dall'altra l'abbattimento totale e ribelle di ogni sorta di identità culturale e valoriale di appartenenza.

Lo scontro che l'uomo di oggi vive è sia esteriore che interiore, un conflitto che si combatte contro il mondo in evoluzione e le sue diversità che interpellano l'umanità e la storia. Come ogni crisi, può da una parte separare fortemente accentuando i lati estremi, dall'altra, essere l'opportunità per recuperare la capacità di vedere l'insieme e ricomprendere il concetto di unità come luogo ove le diversità si donano reciprocamente.

L'ultima cosa da prendere in considerazione è, in ogni caso, quella di far finta di non vedere tale sofferenza, non viverla, lasciarla agli altri o addirittura nasconderla e dimenticarla. Tra i tanti temi approfonditi da Papa Francesco nell'esortazione apostolica Evangelii Gaudium vi è anche quello del conflitto, che se vissuto attraverso l'ottica rispettevole di una comunione di sguardi può dar vita a quella che Zambrano definisce una "seconda nascita". Così scrive il pontefice:

Il conflitto non può essere ignorato o dissimulato. Dev'essere accettato. [...] Di fronte al conflitto, alcuni semplicemente lo guardano e vanno avanti come se nulla fosse, se ne lavano le mani per poter continuare con la loro vita. Altri entrano nel conflitto in modo tale che ne rimangono prigionieri, perdono l'orizzonte, proiettano sulle istituzioni le proprie confusioni e insoddisfazioni e così l'unità diventa impossibile. Vi è però un terzo modo, il più adeguato, di porsi di fronte al conflitto. È accettare di sopportare il conflitto, risolverlo e trasformarlo in un anello di collegamento di un nuovo processo. ${ }^{38}$

Ogni volta che si viene a contatto con una realtà culturale diversa dalla propria, è implicita una certa forma conflittuale sia interiore che esteriore. L'uomo che non si rende cosciente di ciò, togliendosi l'opportunità di interpretare tale conflitto, rischia di escludersi progressivamente dalla relazione con l'altro e bloccare il processo di crescita nel rapporto con sé e con il mondo. L'uomo che, invece, sviluppa una buona capacità relazionale e guarda a questi "conflitti culturali" come un dono di ricchezza, affonda le proprie radici nel mondo e può sentirsi parte di un

38 PAPA FRANCESCO, Evangelii Gaudium. Bologna: Dehoniane, 2013. pp. 152-153. 
processo di evoluzione libero e liberato verso un approdo di reciprocità. Conclude Papa Francesco:

In questo modo, si rende possibile sviluppare una comunione delle differenze, che può essere favorita solo da quelle nobili persone che hanno il coraggio di andare oltre la superficie conflittuale e considerano gli altri nella loro dignità più profonda. Per questo è necessario postulare un principio che è indispensabile per costruire l'amicizia sociale: l'unità è superiore al conflitto. ${ }^{39}$

Per Maria Zambrano ciò che può davvero cambiare la mentalità dell'Occidente e ritracciare uno spazio culturale e sociale percorribile è il delirio. "Delirare", scrive la pensatrice, "è una strana liberazione, la liberazione del soggetto che accetta la vita, cedendole la sua condizione di essere che parla; cedendole la sua logica. [...]. Ogni delirio è figlio della speranza". ${ }^{40}$

La speranza è la prima qualità che devono avere le "nobili perso$n e$ " di cui parla Papa Francesco, speranza che va incarnata in un vivere coraggioso e creativo. Inserirsi nella ferita del conflitto generato dalle diversità risulta decisivo per poter introdurre una cultura della valorizzazione reciproca dei popoli. Scrive Zambrano:

Al pari delle persone, vi sono anche popoli, culture, più aperti al delirio, perché più feriti e quindi più disperatamente aperti alla speranza. Sono essi ad annunciare il futuro non solo per se stessi, giacché la speranza è universale. Vi sono popoli più aperti ad essa e il sentire l'universalità, acutizza, radicalizza e complica il loro delirio. Il loro passato li fa identificare con tutto il passato della storia e il loro avvenire non si delinea con precisione perché è privo dell'immensità del futuro. [...] Sono popoli aperti alla vocazione salvifica della storia: in essi è più ineludibile che altrove far emergere, estrarre la speranza dal delirio, farla entrare nella ragione dinanzi alla verità. ${ }^{41}$

Maria Zambrano con queste parole impregnate della sua esistenza sofferta e delle scoperte relazionali e rivelatrici del suo pensiero, ci chiama a metterci in cammino e a guardare dentro agli "esili del mondo", nei luoghi del "non" e del "senza", dentro le zone sconosciute e dimen-

\footnotetext{
$39 \quad$ Ivi, p. 153.

40 ZAMBRANO, M. L'esilio come patria. Brescia: Morcelliana, 2016. p. 183.

41 Ivi, p. 188.
} 
ticate delle città, nei territori poveri di certezze e privi "dell'immensità del futuro", poiché là ha luogo il delirio, "bagliore albeggiante" di ciò che potrà divenire; là tutto è ancora da scoprire e da sperare, là vi è un foglio ancora bianco che domanda e aspetta di potersi dare alla scrittura.

Ho camminato per le strade di città affollate e meravigliose con il mio originale tra le mani, non levandolo in alto, bensì china, chiedendo perdono per aver osato scrivere un libro. Ma non me ne vergogno; mi commuove aver osato scrivere un libro e sognare una bambina che, come me un tempo, lo conserva in uno scrigno segreto della sua stanza, in attesa di crescere, d'essere grande per poterlo leggere. ${ }^{42}$

Scrivere dell'esilio come luogo è già paradossale se pensiamo che in sé sarebbe definizione di non luogo o luogo nel quale vi è una continua presenza della mancanza, dell'assenza di certezze e di punti fermi attorno al quale costruire la propria vita.

L'esilio è in sé momento di decostruzione, di crollo, di annientamento ed è abitato da una strana "forza di nascita". Per chi lo vive con la tenacia e la fedeltà di Maria Zambrano diviene un "pozzo di rivelazioni", un "serbatoio" immenso di vita dal quale attingere per scrivere e ripensare la propria storia.

La porta di accesso all'esilio è quella dell'abbandono, condizione del non essere che si inserisce tra il vivere e il morire, dove vita e morte vengono entrambe rinviate. Soltanto immergendo lo sguardo da questa prospettiva è stato possibile, in parte, avvicinare l'immagine dell'esiliato che la Zambrano rivela come un essere umano rimasto senza luogo. L'esperienza di questo "vuoto spaziale", del fallimento continuo, delle difficoltà concrete e della "lontananza del conosciuto", intesa come lontananza dall'essere se stessi inseriti nella propria vita, sono tutte realtà impossibili da comprendere se non vissute in prima persona. Su questo preciso fattore, di "imprecisato esistere", Maria Zambrano si mette in cammino e comincia la costruzione del suo pensiero. Potremmo dire che fa della sua condanna la rinascita, della maledizione una benedizione, del paradosso la sua vocazione. Da questa prospettiva l'esilio è la sua patria, poche esperienze infatti possono essere a tal punto rivelatrici da penetrare radicalmente e fondare il pensiero di un filosofo. In Maria Zambrano l'esilio è fondante, è la pietra preziosa che custodisce i segreti

42 ZAMBRANO, M. Le parole del ritorno. Enna: Città Aperta, 2003. p. 130. 
di un'esistenza intera ed è da lì che ogni volta bisogna partire per provare a relazionarsi con lei.

"L'esilio è, dunque creatore" ${ }^{43}$ scrive Zambrano. Lo è per le rivelazioni che ha vissuto e che ancora stiamo cercando di comprendere ma anche per quelle che si celano nella misteriosa storia del mondo. Attraverso l'esilio la pensatrice ci contagia nel desiderio di conoscere, di penetrare la nostra epoca nelle domande più grandi, di non affievolire mai la nostra ricerca innovativa nelle terre che ospitano grandi promesse, portate soprattutto da ferite e diversità. L'esilio è in sé il viaggio di sofferenza e rivelazione che ognuno di noi deve affrontare per giungere alla patria, la quale, appunto, sta sempre oltre un esilio.

Maria Zambrano giunge ad amare questo luogo e riconoscerlo come la sua patria perché lo accetta, si rende conto che a lei è stato chiesto di vivere un tale abbandono e non se lo tiene per sé, ma lo vive in una dinamica di apertura con gli altri: con i suoi studenti nelle lezioni, con il pubblico nelle conferenze, con i lettori nella scrittura. L'esilio le si porge così sotto una nuova luce, quella di uno spazio dove si rigenera la costante opportunità creativa e delirante di dare una speranza di unità al mondo.

Il "nulla" accettato e assunto dalla Zambrano è il "vuoto fecondo" nel quale può accendersi l'esperienza dell'unità, è la meraviglia di riconoscersi e porgersi come dono all'umanità. Il pensatore Zanghì scrive che questo misterioso donarsi è:

l'atto nel quale la creatura umana si apre, nell'unità di tutte le sue componenti, per accogliere e darsi. Accogliere nella luce il grande mare dell'essere; darsi nella luce alle sue immense acque. Un uscire dall'intimo, dal segreto, per essere. Questo aprirsi che è il pensare, di fatto è creativo, è generativo: esiste nella parola, nella quale il pensiero esce come da se stesso, silenzio luminoso, per essere, dandosi forma: facendosi conoscenza. ${ }^{44}$

\section{Conclusioni}

Giunti al termine di questo lavoro sembra opportuno evidenziare quelli che possono essere i potenziali frutti di una ricerca che necessiterebbe di ulteriori sviluppi e approfondimenti.

43 ZAMBRANO, M. L'esilio come patria. Brescia: Morcelliana, 2016. p. 142.

44 ZANGHì, G.M. Leggendo un carisma. Chiara Lubich e la cultura. Roma: Città Nuova, 2015. p. 62. 
Pensare ciò che vive, coincide nella filosofa spagnola, con lo scrivere ciò che sente e tale particolarità è uno degli elementi essenziali nella filosofia zambraniana. L'autrice converte l'esilio nel luogo prediletto del pensare e dello scrivere, distinguendo ogni volta i tratti personali e quelli universali.

Ma quando parliamo di esilio non affrontiamo solo il tema degli esiliati politici. Per cogliere le rivelazioni di Maria Zambrano è necessario fare un passo successivo e interpretare l'esilio come il momento di perdita, di smarrimento personale, di malattia, di solitudine, ogni volta, cioè, che l'uomo varca la porta d'accesso al territorio sconosciuto dell'abbandono: quella condizione del non essere che si inserisce tra il vivere e il morire, dove vita e morte vengono entrambe rinviate.

Immergendo lo sguardo da questa prospettiva è stato possibile, in parte, avvicinare l'immagine dell' esiliato che la Zambrano rivela come un essere umano rimasto senza luogo. L'esperienza di questo "vuoto spaziale", del fallimento continuo, delle difficoltà concrete e della "lontananza del conosciuto", intesa come lontananza dall'essere se stessi inseriti nella propria vita, sono tutte realtà impossibili da comprendere se non vissute in prima persona. Su questo preciso fattore, di "imprecisato esistere", Maria Zambrano si mette in cammino e comincia la costruzione del suo pensiero. Potremmo dire che fa della sua condanna la rinascita, della maledizione una benedizione, del paradosso la sua vocazione. Da questa prospettiva l'esilio è la sua patria, poche esperienze infatti possono essere a tal punto rivelatrici da penetrare radicalmente e fondare il pensiero di un filosofo. In Maria Zambrano l'esilio è fondante, è la pietra preziosa che custodisce i segreti di un'esistenza intera ed è da lì che ogni volta bisogna partire per provare a relazionarsi con lei.

Se la porta d'accesso è l'abbandono, il dolore del sentirsi lontani dalla propria patria e dal proprio modo di vivere, allora la lettura dello spazio dell'esilio può divenire una lettura anche culturale e interculturale. Abbiamo visto come altri pensatori ed esponenti religiosi abbiano, negli anni, espresso il pensiero di una rinascita sociale che coinvolga sia la sfera intellettuale che quella esistenziale dell'uomo, poiché là dove la diversità viene accolta e valorizzata, l'uomo è capace di trasformare un problema oggettivo in una nuova risorsa per il proprio paese e per l'umanità.

Guardando a questo tipo di esperienza testimoniata in maniera egregia da Maria Zambrano nell'arco della sua vita, non possiamo esimerci dal raccogliere la sua sfida culturale con la speranza creativa di 
chi desidera risolvere il problema, la ferita, il conflitto per «trasformarlo in un anello di collegamento di un nuovo processo» ${ }^{45}$.

\section{Riferimenti bibliografici}

CACCIARI, M. L'arcipelago. Milano: Adelphi, 1997.

JALLADE, S. Il richiamo della strada. Portogruaro: Ediciclo, 2011.

LEVINAS, E. Dall'esistenza all'esistente. Genova: Marietti, 2000.

LUBICH, Ch. Colloqui con i gen. Roma: Città Nuova, 1974.

O'CONNOR, F. Nel territorio del diavolo, sul mistero di scrivere. Roma: Minimun Fax, 2010.

PAPA FRANCESCO. Evangelii Gaudium. Bologna: Dehoniane, 2013. ZAMBRANO M. Lettera sull'esilio. In: Cuadernos del Congreso por la libertad de la cultura, 49 (1961), pp. 65-70.

. Senderos. Barcellona: Anthropos, 1986.

. Delirio y destino. Madrid: Mondadori, 1989.

. Verso un sapere dell'anima. Milano: Raffaello Cortina, 1996.

. Le parole del ritorno. Enna: Città Aperta, 2003. . I Beati, Milano: SE SRL, 2010.

. L'esilio come patria, Brescia: Morcelliana, 2016.

ZANGHİ, G.M. Leggendo un carisma. Chiara Lubich e la cultura. Roma: Città Nuova, 2015.

45 Papa Francesco, Evangelii Gaudium, Bologna: Dehoniane, 2013, pp. 152-153 